\title{
EXCITATION OF SHEAR WAVES IN A PIEZOCERAMIC MEDIUM WITH PARTIALLY ELECTRODIZED TUNNEL OPENINGS STRENGTHENED BY A RIGID STRINGER
}

\author{
D. I. BARDZOKAS AND G. I. SFYRIS
}

Received 2 May 2003

An antiplane mixed boundary electroelasticity of a stationary wave process in an unbounded piezoceramic medium containing tunnel heterogeneities of opening or thin rigid inclusion (stringer) type is considered. The excitation of an electric field occurs at the expense of differences of electric potentials applied to the system of electrodes located on a free from stresses opening surface. Using the correct integral representations of the solutions, the boundary problem is reduced to the system of singular integrodifferential equations of the second type with resolvent kernels. The results of the parametric investigations characterizing the behavior of the components of an electroelastic field in the medium area and on the opening surface are given. A system of singular integrodifferential equations is obtained for investigation of a conjugated electroelastic field in a piezomedium with a tunnel along the material axis opening a rigid curvilinear inclusion, excited by a system of active electrodes, located on the opening surface. The solvable system of equations of the boundary problem is reduced to two differential equations of Helmhöltz and Laplace with respect to the amplitude of shear displacement and some auxiliary functions. The obtained system is solved numerically by a special scheme of the method of quadrature.

\section{Introduction}

The analysis of interaction of surface electrodes with piezoceramic material in the theory of piezoceramic transformations and on various devices on the surfaces of waves must be carried out on the basis of the strict theory of electroelasticity $[8,10,11]$ and the methods of solution of boundary problems with resolvent conditions on the boundary.

As it is known $[3,7]$, the edges of the electrodes serve as the sources of concentration of electric and mechanical fields, and it means that in these areas there may appear microcracks or develop a breakdown. The criterion of an electromechanical failure of piezoelectrics initiated by the edges of the electrodes is suggested in [3]. In [4], there is considered a problem of excitation of Rayleigh waves in a halfspace with the finite system of electrodes. The plane static problems of the theory of elasticity for plates containing 
the defects of stringer or crack type were considered, for example, in [1, 12]. Some static and dynamic antiplane problems for a circular cylinder with one or two symmetrically located electrodes by the method of series are solved in [11]. The approach, based on the method of boundary integral equations, to the solution of an antiplane problem of oscillations of a homogeneous piezoceramic cylinder excited by the arbitrary system of electrodes is suggested in [6]. In [2], there are considered some dynamic problems of electroelasticity for infinite piezoceramic cylinders with linear defects of tunnel crack and inclusion type susceptible to the harmonic with time mechanical loading.

In the given article there is obtained the system of singular integrodifferential equations for investigation of a conjugated electroelastic field in a piezomedium with a tunnel along the material symmetry axis opening and a rigid curvilinear inclusion, excited by a system of active electrodes, located on the opening surface. It is assumed that on the electrodes there are prescribed the harmonically changing with time differences of electric potentials, and the unelectrodized areas of the opening surface come in contact with vacuum (air). The solvable system of equations of the boundary problem is reduced to two differential equations of Helmhöltz and Laplace with respect to the amplitude of shear displacement and some auxiliary function. To solve this problem there are constructed integral representations of the solutions, the substitution of the limiting values of which into boundary condition brings to the system of singular integrodifferential equations of the second type with resolvent kernels. The obtained system is solved numerically by a special scheme of the method of quadrature. Results of the numerical investigations are given.

\section{Formulation of the problem}

In Cartesian coordinates $O x_{1} x_{2} x_{3}$ consider a continuous piezoceramic medium containing a tunnel opening and thin rigid $L$. The cross section of the opening is bounded with sufficiently smooth contour $C$. On the free from the mechanical stresses opening surface there are placed $2 l$ infinitely long in the direction of axis $x_{3}$ thin electrodes with given differences of the electric potential. The unelectrodized sections of the opening surface are bounded with vacuum. The boundaries of $k$ th electrode are determined by quantities $\beta_{2 k-1}$ and $\beta_{2 k}(k=\overline{1,2 \ell})$, and the electric potential on them is prescribed by quantity $\phi_{k}^{*}=\operatorname{Re}\left(\Phi_{k}^{*} e^{-i \omega t}\right.$ ) ( $\omega$ is the circular frequency, $t$ is the time). It is assumed that axis $x_{3}$ coincides with the direction of the electric field of a preliminary polarization of the piezoceramics, and the electrodes are weightless and have negligible rigidity. The location of the electrodes, the relative position, and configuration of heterogeneities cannot be quite arbitrary; the conditional matching imposed on them will be indicated below.

In the given conditions, in a piecewise-homogeneous medium, there occur an electroelastic field corresponding to the state of antiplane deformation. The full system of equations in the quasistatic approximation includes the following relations [11]:

(i) equations of movement:

$$
\partial_{1} \sigma_{13}+\partial_{2} \sigma_{23}=\rho \frac{\partial^{2} u_{3}}{\partial t^{2}}, \quad \partial_{i}=\frac{\partial}{\partial x_{i}}
$$


(ii) constitutive equations of the medium:

$$
\begin{gathered}
\sigma_{m 3}=c_{44}^{E} \partial_{m} u_{3}-e_{15} E_{m}, \\
D_{m}=e_{15} \partial_{m} u_{3}+\jmath_{11}^{\varepsilon} E_{m} \quad(m=1,2),
\end{gathered}
$$

(iii) equations of the electrostatics:

$$
\operatorname{div} \mathbf{D}=0, \quad \mathbf{E}=-\operatorname{grad} \phi .
$$

In (2.1), (2.2), and (2.3), $\sigma_{m 3}(m=1,2)$ are the components of the stress tensor, $u_{3}$ is the component of the elastic displacement vector, $\mathbf{E}$ and $\mathbf{D}$ are the vectors of the strength and induction of an electric field; $\phi$ is the electric potential; $c_{44}^{E}, e_{15}$ and $\ni_{11}^{\varepsilon}$ are the shear modulus measured at a constant value of the electric field, piezoelectric constant and dielectric permittivity measured at fixed deformations, respectively, $\rho$ is the mass density of the material.

The system of (2.1), (2.2), and (2.3) is reduced to the differential equations with respect to displacement $u_{3}$ and electric potential $\phi$,

$$
\begin{gathered}
c_{44}^{E} \nabla^{2} u_{3}+e_{15} \nabla^{2} \phi=\rho \frac{\partial^{2} u_{3}}{\partial t^{2}}, \\
e_{15} \nabla^{2} u_{3}-\ni_{11}^{\varepsilon} \nabla^{2} \phi=0 .
\end{gathered}
$$

From (2.4) follow the relations

$$
\begin{gathered}
\nabla^{2} u_{3}-c^{-2} \frac{\partial^{2} u_{3}}{\partial t^{2}}=0, \quad \nabla^{2} F=0, \\
\phi=\frac{e_{15}}{\vartheta_{11}^{\varepsilon}} u_{3}+F, \quad c=\sqrt{\frac{c_{44}^{E}\left(1+k_{15}^{2}\right)}{\rho}}, \quad k_{15}=\frac{e_{15}}{\sqrt{c_{44}^{E} \ni_{11}^{\varepsilon}}},
\end{gathered}
$$

where $c$ is the velocity of the shear wave in a piezoelectric medium, $k_{15}$ is the factor of electromechanic connection [11].

The mechanical and electric quantities allowing for (2.2), (2.3), and (2.5) may be expressed as functions $u_{3}$ and $F$ by the formulas [2]

$$
\begin{gathered}
\sigma_{13}-i \sigma_{23}=2 \frac{\partial}{\partial z}\left[c_{44}^{E}\left(1+k_{15}^{2}\right) u_{3}+e_{15} F\right], \\
D_{1}-i D_{2}=-2 \ni_{11}^{\varepsilon} \frac{\partial F}{\partial z}, \quad E_{1}-i E_{2}=-2 \frac{\partial}{\partial z}\left(F+\frac{e_{15}}{\vartheta_{11}^{\varepsilon}} u_{3}\right), \quad z=x_{1}+i x_{2} .
\end{gathered}
$$

Assuming $u_{3}=\operatorname{Re}\left(U_{3} e^{-i \omega t}\right), \phi=\operatorname{Re}\left(\Phi e^{-i \omega t}\right)$, and $F=\operatorname{Re}\left(e^{-i \omega t} F^{*}\right)$, we will write (2.5) relating to amplitude quantities

$$
\begin{array}{cc}
\nabla^{2} U_{3}+\gamma^{2} U_{3}=0, & \nabla^{2} F^{*}=0, \\
\Phi=\frac{e_{15}}{\vartheta_{11}^{\varepsilon}} U_{3}+F^{*}, & \gamma=\frac{\omega}{c},
\end{array}
$$

where $\gamma$ is the wave number. 
234 Excitation of shear waves in a piezoceramic medium

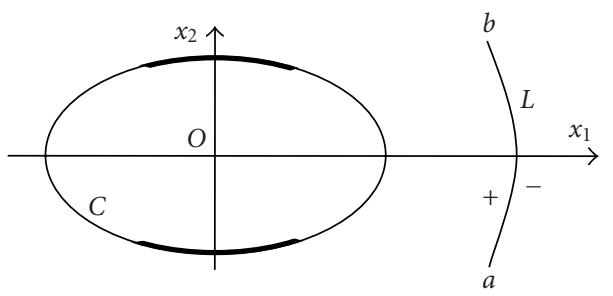

Figure 2.1. A medium with a partially electrodized opening and curvilinear stringer.

Considering that the stringer is attached we represent the mechanical and electric boundary conditions on the contour $L$ in the following way:

$$
\begin{gathered}
u_{3}^{ \pm}=0, \\
E_{s}^{+}=E_{s}^{-}, \quad D_{n}^{+}=D_{n}^{-} .
\end{gathered}
$$

Here $E_{s}$ and $D_{n}$ are the tangential component of the vector of electric strength and normal component of the vector of electric induction, respectively, the signs "plus" and "minus" refer to the left and right edges of inclusion $L$ when moving from its beginning $a$ to the end $b$ (Figure 2.1).

To obtain the efficient from the point of view of the numerical realization system of integral equations, it is advisable to differentiate the boundary condition (2.8) over the $\operatorname{arc}$ abscissa $s$,

$$
\left(\frac{\partial u_{3}}{\partial s}\right)^{ \pm}=0
$$

The mathematical record of the boundary conditions on an opening surface allowing for (2.6) has the following form:

$$
\begin{gathered}
\frac{\partial}{\partial n}\left\{c_{44}^{E}\left(1+k_{15}^{2}\right) u_{3}+e_{15} F\right\}=0, \\
\phi=F+\frac{e_{15}}{\jmath_{11}^{\varepsilon}} u_{3}=\phi^{*}(\zeta, t), \quad \zeta \in C_{\phi}, \\
D_{n}=-\ni_{11}^{\varepsilon} \frac{\partial F}{\partial n}=0, \quad C \backslash C_{\phi},
\end{gathered}
$$

where $C_{\phi}$ is a part of contour $C$, corresponding to an electrodized cavity surface, differential operator $\partial / \partial n$ denotes the derivative with respect to normal to contour $C$.

Thus, the problem is in the definition of functions $U_{3}$ and $F^{*}$ from (2.7) and boundary conditions (2.9), (2.10), (2.11), (2.12), and (2.13). 


\section{The system of singular integrodifferential equations of a boundary problem of electroelasticity}

To bring the considered boundary problem of electroelasticity to integral equations, we present the amplitude of sought-for functions $u_{3}$ and $F$ in the following form [2]:

$$
\begin{gathered}
U_{3}\left(x_{1}, x_{2}\right)=\frac{i}{4 c_{44}^{E}\left(1+k_{15}^{2}\right)}\left\{\int_{L} q(\zeta) H_{0}^{(1)}(\gamma r) d s+\int_{C} p\left(\zeta^{*}\right) H_{0}^{(1)}\left(\gamma r_{1}\right) d s\right\}, \\
F^{*}\left(x_{1}, x_{2}\right)=-\frac{1}{2 \pi \ni_{11}^{\varepsilon}} \int_{C} f\left(\zeta^{*}\right) \frac{\partial}{\partial n} \ln r_{1} d s, \\
r=|\zeta-z|, \quad r_{1}=\left|\zeta^{*}-z\right|, \quad \zeta \in L, \zeta^{*} \in C .
\end{gathered}
$$

Here $H_{v}^{(1)}(x)$ is the Hankel function of the first kind of $v$ order, $d s$ is the element of the contour arc length over which the integration is carried out.

Integral representations (3.1) satisfy differential equations (2.7) and electric conditions (2.9) on inclusion $L$ and also provide the fulfillment of condition $\left[U_{3}\right]=U_{3}^{+}-U_{3}^{-}=0$ in (2.8).

Substituting the limiting values of function (3.1) and their derivatives at $z \rightarrow \zeta_{0} \in L$ and $z \rightarrow \zeta_{0}^{*} \in C$ in boundary conditions (2.10), (2.11), (2.12), and (2.13), we arrive at the system of integrodifferential equations of the second kind:

$$
\begin{aligned}
& \int_{L} q(\zeta) G_{1}\left(\zeta, \zeta_{0}\right) d s+\int_{C} p\left(\zeta^{*}\right) G_{2}\left(\zeta^{*}, \zeta_{0}\right) d s=0, \quad \zeta_{0} \in L, \\
& \int_{L} q(\zeta) G_{3}\left(\zeta, \zeta_{0}^{*}\right) d s+\int_{C} p\left(\zeta^{*}\right) G_{4}\left(\zeta^{*}, \zeta_{0}^{*}\right) d s \\
& \quad+\int_{C} f^{\prime}\left(\zeta^{*}\right) G_{5}\left(\zeta^{*}, \zeta_{0}^{*}\right) d s-2 p\left(\zeta_{0}^{*}\right)=0, \quad \zeta_{0}^{*} \in C, \\
& \int_{C} f^{\prime}\left(\zeta^{*}\right) G_{6}\left(\zeta^{*}, \zeta_{0}^{*}\right) d s=0, \quad \zeta_{0}^{*} \in C \backslash C_{\phi}, \\
& \int_{L} q(\zeta) G_{7}\left(\zeta, \zeta_{0}^{*}\right) d s+\int_{C} p\left(\zeta^{*}\right) G_{8}\left(\zeta^{*}, \zeta_{0}^{*}\right) d s \\
& \quad+\int_{C} f\left(\zeta^{*}\right) G_{9}\left(\zeta^{*}, \zeta_{0}^{*}\right) d s+\frac{1}{2 \vartheta_{11}^{\varepsilon}} f\left(\zeta_{0}^{*}\right)=\Phi\left(\zeta_{0}^{*}\right), \quad \zeta_{0}^{*} \in C_{\phi},
\end{aligned}
$$

in which kernels $G_{m}(m=1,9)$ are determined by the following expressions:

$$
\begin{gathered}
G_{1}\left(\zeta, \zeta_{0}\right)=\gamma H_{1}\left(\gamma r_{0}\right) \sin \left(\psi_{0}-\alpha_{0}\right)-\frac{2 i}{\pi} \operatorname{Im} \frac{e^{i \psi_{0}}}{\zeta-\zeta_{0}}, \\
G_{2}\left(\zeta^{*}, \zeta_{0}\right)=\gamma H_{1}^{(1)}\left(\gamma r_{10}\right) \sin \left(\psi_{0}-\alpha_{10}\right), \\
G_{3}\left(\zeta, \zeta_{0}^{*}\right)=i \gamma H_{1}^{(1)}\left(\gamma r_{20}\right) \cos \left(\psi_{10}-\alpha_{20}\right), \\
G_{4}\left(\zeta^{*}, \zeta_{0}^{*}\right)=i \gamma H_{1}\left(\gamma r_{30}\right) \cos \left(\psi_{10}-\alpha_{30}\right)+\frac{2}{\pi} \operatorname{Re} \frac{e^{i \psi_{10}}}{\zeta^{*}-\zeta_{0}^{*}},
\end{gathered}
$$




$$
\begin{gathered}
G_{5}\left(\zeta^{*}, \zeta_{0}^{*}\right)=-\frac{2 e_{15}}{\pi \jmath_{11}^{\varepsilon}} \operatorname{Im} \frac{e^{i \psi_{10}}}{\zeta^{*}-\zeta_{0}^{*}}, \\
G_{6}\left(\zeta^{*}, \zeta_{0}^{*}\right)=\operatorname{Im} \frac{e^{i \psi_{10}}}{\zeta^{*}-\zeta_{0}^{*}}, \\
G_{7}\left(\zeta, \zeta_{0}^{*}\right)=\frac{i k_{15}^{2}}{4 e_{15}\left(1+k_{15}^{2}\right)} H_{0}^{(1)}\left(\gamma r_{20}\right), \\
G_{8}\left(\zeta^{*}, \zeta_{0}^{*}\right)=\frac{i k_{15}^{2}}{4 e_{15}\left(1+k_{15}^{2}\right)} H_{0}^{(1)}\left(\gamma r_{30}\right), \\
G_{9}\left(\zeta^{*}, \zeta_{0}^{*}\right)=-\frac{1}{2 \pi \jmath_{11}^{\varepsilon}} \operatorname{Re} \frac{e^{i \psi_{1}}}{\zeta^{*}-\zeta_{0}^{*}}, \\
H_{1}(x)=\frac{2 i}{\pi x}+H_{1}^{(1)}(x), \quad r_{0}=\left|\zeta-\zeta_{0}\right|, \quad r_{10}=\left|\zeta^{*}-\zeta_{0}\right|, \\
r_{20}=\left|\zeta-\zeta_{0}^{*}\right|, \quad r_{30}=\left|\zeta^{*}-\zeta_{0}^{*}\right|, \quad \alpha_{0}=\arg \left(\zeta-\zeta_{0}\right), \\
\alpha_{10}=\arg \left(\zeta^{*}-\zeta_{0}\right), \quad \alpha_{20}=\arg \left(\zeta-\zeta_{0}^{*}\right), \quad \alpha_{30}=\arg \left(\zeta^{*}-\zeta_{0}^{*}\right), \\
\psi_{0}=\psi\left(\zeta_{0}\right), \quad \psi_{10}=\psi_{1}\left(\zeta_{0}^{*}\right) ; \quad \zeta, \zeta_{0} \in L ; \zeta^{*}, \zeta_{0}^{*} \in C .
\end{gathered}
$$

Here $\psi$ and $\psi_{1}$ are the angles between the normals to contours $L$ and $C$ and axis $O x_{1}$, respectively; $\Phi\left(\zeta_{0}^{*}\right)$ is the piecewise constant function assigning the values of the amplitude of electric potentials on the electrodized sections of an opening surface.

It should be noted here that as the appearing in the process of oscillation reflected from inclusions electroelastic waves introduce additional charges on paired (powered by a separate generator) electrodes, the position of the latter, and also the relative position of the opening and inclusion and their configuration should provide the similarity of the additional charges (over the absolute value). Otherwise the system (3.2) becomes unsolvable.

\section{Intensity of interaction contact forces of an inclusion and a piezomedium}

Consider the behavior of an electroelastic field in the vicinity of an inclusion. From integral representation (3.1) for a displacement amplitude there follows the following equality:

$$
q(\zeta)=c_{44}^{E}\left(1+k_{15}^{2}\right)\left[\frac{\partial U_{3}}{\partial n}\right]
$$

where the square brackets denote the corresponding values on $L$. From equations of state (2.2) and relations (2.7), (2.9) it follows that

$$
\begin{gathered}
\sigma_{n}=\operatorname{Re}\left(T_{n} e^{-i \omega t}\right) \\
{\left[T_{n}\right]=c_{44}^{E}\left[\frac{\partial U_{3}}{\partial n}\right]+e_{15}\left[\frac{\partial \Phi}{\partial n}\right], \quad\left[\frac{\partial \Phi}{\partial n}\right]=\frac{e_{15}}{\vartheta_{11}^{\varepsilon}}\left[\frac{\partial U_{3}}{\partial n}\right] .}
\end{gathered}
$$


From expressions (4.1), (4.2), we obtain the equation

$$
q(\zeta)=\left[T_{n}\right]
$$

Thus, on the basis of (4.3) function $q(\zeta)$ may be interpreted as the intensity of interaction contact forces of a rigid inclusion and a piezomedium. Therefore, to attain the equilibrium of the inclusion, the following equality must be satisfied:

$$
\int_{L} q(\zeta) d s=0
$$

By asymptotic analysis of singular integral equations in the vicinity of the inclusion tip on the line of integration it may be shown that on the ends of $L$ function $q(\zeta)$ possesses singularities of root type. Thus, condition (4.4) must be considered as additional for solution of the system of singular integrodifferential equations (3.2) in the class of functions not restricted at the ends of $L[9]$.

Due to (2.2), (2.9), and (2.10), we have

$$
\left[D_{s}^{*}\right]=\vartheta_{11}^{\varepsilon}\left[E_{s}^{*}\right]=0, \quad\left[E_{n}^{*}\right]=-\frac{e_{15}}{\vartheta_{11}^{\varepsilon}}\left[\frac{\partial U_{3}}{\partial n}\right]=-\frac{k_{15}^{2}}{\left(1+k_{15}^{2}\right) e_{15}} q(\zeta) .
$$

On the basis of (4.5) we may come to a conclusion that the vector of electric induction D is continued over $L$, and the vector of electric $\mathbf{E}$ undergoes a breakage on the inclusion.

Defining the functions $q(\zeta), P\left(\zeta^{*}\right)$, and $f\left(\zeta^{*}\right)$ by formulas $(2.5),(2.6)$ and applying representations (3.1), we may calculate all the components of the wave electroelastic field in a piecewise homogeneous medium.

We find, for example, the amplitude of density distribution of electric charges $\rho_{k}(\beta)$ on $k$ th electrode. Introducing the parametrization of the contour $C$ with the help of equalities $\zeta^{*}=\zeta^{*}(\beta), \zeta_{0}^{*}=\zeta^{*}\left(\beta_{0}\right)\left(0 \leq \beta, \beta_{0} \leq 2 \pi\right)$ and taking into account that the opening is connected with vacuum, we may write down [2]

$$
\rho_{k}(\beta)=D_{n}^{(k)}(\beta), \quad \alpha_{2 k-1}<\beta<\alpha_{2 k} .
$$

Here, $D_{n}^{(k)}(\beta)$ represents the amplitude of normal component of the electric induction vector on the corresponding area of contour $C$ covered with electrodes. Applying integral representation (3.1) of the function $F^{*}\left(x_{1}, x_{2}\right)$ and allowing for equalities (2.13), (4.6), we find

$$
\rho_{k}\left(\beta_{0}\right)=-\ni_{11}^{\varepsilon} \int_{C} f^{\prime}\left(\zeta^{*}\right) \operatorname{Im} \frac{e^{i \psi_{10}}}{\zeta^{*}-\zeta_{0}^{*}} d s, \quad \zeta_{0}^{*} \in C_{\phi_{k}} .
$$

Here $C_{\phi_{k}}$ is a part of the contour $C$ where $k$ th electrode is placed.

Integrating expression (4.7) by variable $\beta_{0}$ in the limits from $\beta_{2 k-1}$ to $\beta_{2 k}$, we obtain the peak value of the total charge of the $k$ th electrode relating to its length unity. The current flowing through the given electrode and equal to the conduction current in the generator circuit may be defined by the formulas

$$
I_{k}(t)=\operatorname{Re}\left\{i \omega e^{-i \omega t} \int_{\alpha_{2 k-1}}^{\alpha_{2 k}} \rho_{k}\left(\beta_{0}\right) d \beta_{0}\right\}, \quad s^{\prime}\left(\beta_{0}\right)=\frac{d s}{d \beta_{0}} .
$$


238 Excitation of shear waves in a piezoceramic medium

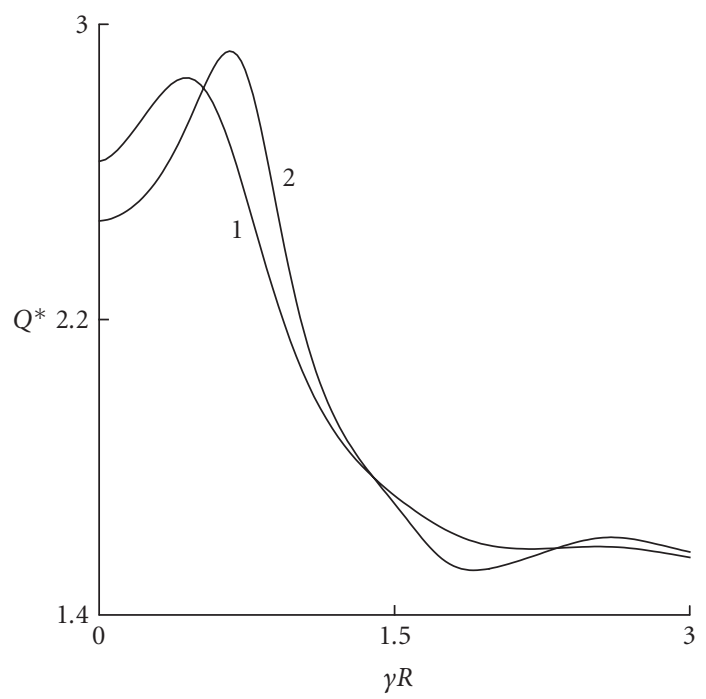

Figure 5.1. Changes of a relative total electric charge on an electrode in the function of the normalized wave number $\left(h / R_{1}=1.5\right)$.

\section{Definition of the concentration of stresses in the vincinity of an opening}

To define the concentration of shear stresses near the opening we should calculate stress $\sigma_{s}=\sigma_{23} \cos \psi_{1}-\sigma_{13} \sin \psi_{1}$ on its surface. Taking into account (2.2), (2.3), and (2.5), we will have

$$
\sigma_{s}=\operatorname{Re}\left(T_{s} e^{-i \omega t}\right), \quad T_{s}\left(\zeta_{0}^{*}\right)=c_{44}^{E}\left(1+k_{15}^{2}\right) \frac{\partial U_{3}}{\partial s}+e_{15} \frac{\partial F^{*}}{\partial s}
$$

Here by partial derivatives from the corresponding quantities we imply their restricting values at $z \rightarrow \zeta_{0}^{*}$ from the area of the body. Proceeding from (5.1) and representations (3.1), we find

$$
\begin{aligned}
T_{s}\left(\zeta_{0}^{*}\right)= & \int_{L} q(\zeta) g_{1}\left(\zeta, \zeta_{0}^{*}\right) d s+\int_{C} p\left(\zeta^{*}\right) g_{2}\left(\zeta^{*}, \zeta_{0}^{*}\right) d s \\
& +\int_{C} f^{\prime}\left(\zeta^{*}\right) g_{3}\left(\zeta^{*}, \zeta_{0}^{*}\right) d s+\frac{e_{15}}{2 \ni_{11}^{\varepsilon}} f^{\prime}\left(\zeta_{0}^{*}\right), \\
g_{1}\left(\zeta, \zeta_{0}^{*}\right)= & -\frac{i \gamma}{4} H_{1}^{(1)}\left(\gamma r_{20}\right) \sin \left(\psi_{10}-\alpha_{20}\right), \\
g_{2}\left(\zeta^{*}, \zeta_{0}^{*}\right)= & -\frac{i \gamma}{4} H_{1}^{(1)}\left(\gamma r_{30}\right) \sin \left(\psi_{10}-\alpha_{30}\right), \\
g_{3}\left(\zeta^{*}, \zeta_{0}^{*}\right)= & -\frac{e_{15}}{2 \pi \ni_{11}^{\varepsilon}} \operatorname{Re} \frac{e^{i \psi_{10}}}{\zeta *-\zeta_{0}^{*}} .
\end{aligned}
$$

The above quantities $\psi_{10}, r_{20}, r_{30}, \alpha_{20}, \alpha_{30}$ are determined in (3.2).

Formula (5.2) allows investigating the concentration of stresses according to the frequency of harmonic excitation, configuration of the cross section of the opening and 
inclusion, quantity and position of the active surface electrodes and the insert, quantities and locations of active surface electrodes.

\section{Examples of calculations}

Consider a piezoceramic space (material PZT-4 [5]) containing an opening of elliptic cross section and an inclusion, the contour of which is parabolic. The parametric equations of contours $L$ and $C$ have the following forms, respectively:

$$
\begin{aligned}
\zeta & =\delta e^{i \vartheta}\left(p_{1}+i p_{2} \delta\right)+h, \quad \delta \in[-1,1], \\
\zeta^{*} & =R_{1} \cos \beta+i R_{2} \sin \beta, \quad \beta \in[0,2 \pi],
\end{aligned}
$$

where $\vartheta$ is the angle characterizing the orientation of the stringer in a space.

Assume that two surface electrodes carry out the excitation of the medium with difference of the amplitude of potentials $2 \Phi^{*}$, the centers of which lie on axis $x_{2}\left(\beta_{1}=\right.$ $\left.5 \pi / 14, \beta_{2}=9 \pi / 14, \beta_{3}=19 \pi / 14, \beta_{4}=23 \pi / 14\right)$.

Solution of the system of integrodifferential equations (3.2) together with (4.4) allowing for (6.1) was carried out by the scheme of the method of quadratures [2].

Investigate the influence of the dynamic effect on the behavior of the total electric charge on an electrode in case of an opening interacting with linear stringer $\left(p_{2} / R_{1}=0\right)$. Figure 5.1 shows the behavior of quantity $Q^{*}=\left|Q /\left(\ni_{11}^{\varepsilon} \Phi^{*}\right)\right|$ ( $Q$ is the total electric charge on an electrode) in the function of normalized wave number $\gamma R$. Curves 1 and 2 are constructed for values of parameters $R_{2} / R_{1}=1, h / R_{1}=1.5, \vartheta=\pi / 2, p_{1} / R_{1}=1$, and 3 for $\left(R=0.5\left(R_{1}+R_{2}\right)\right)$, respectively. The similar graphs for the same parameters, except $h / R_{1}=3$, are represented in Figure 6.1. The dashed line here relates to the case when there is no inclusion. Quantity $Q$ has been calculated with the help of formula (4.7).

Investigate the intensity of contact forces on an insert according to different parameters. Figure 6.2 represents the changing of quantity $\lambda=\left|q(\delta) / \Phi^{*}\right|$ due to the given on two surface electrodes difference of potentials $2 \Phi^{*}$. The calculation is carried out on the elliptic opening $\left(R_{1} / R_{2}=2\right)$ with linear inclusion at $p_{1} / R_{1}=2, h / R_{1}=1.5, \vartheta=\pi / 2$. Curves $1-4$ are constructed for values $\gamma R=0,1,2$, and 3, respectively.

Changing of $\lambda$ on the contour of a parabolic inclusion is illustrated in Figure 6.3 by curves $1,2,3$ for values $p_{2} / R_{1}=0,1.5,3$, respectively, at $\gamma R=0.6, R_{1} / R_{2}=2, p_{1} / R_{1}=3$, $h / R_{1}=2.5, \vartheta=\pi / 2$.

In Figures 6.4 and 6.5 the data of calculations characterizing the distribution of quantity $\eta=c_{44}^{E}\left|U_{3} / \Phi^{*}\right|$ are represented on the contour of an opening with linear and parabolic inclusion, respectively. Curves $1-4$ in Figure 6.4 are constructed for the values of normalized wave number $\gamma R=0,1,2$, and 3 at $R_{1} / R_{2}=2, p_{2} / R_{1}=2, h / R_{1}=1.5, \vartheta=\pi / 2$. Curves $1-3$ in Figure 6.5 correspond to values $p_{1} / R_{1}=3, R_{1} / R_{2}=2, h / R_{1}=2.5, \gamma R=0.6$, $\vartheta=\pi / 2, p_{2} / R_{1}=0,1.5$, and 3 .

It should be noted here that when $\vartheta=0$ the linear insert at the given symmetric position of two electrodes does not cause disturbance in the electroelastic state of the medium with an opening. The calculations show that in that case $q(\zeta) \equiv 0$.

Investigation of the behavior of mechanical quantities at an electric excitation of a piecewise homogeneous medium is of great interest. The lines of the equal modulus 


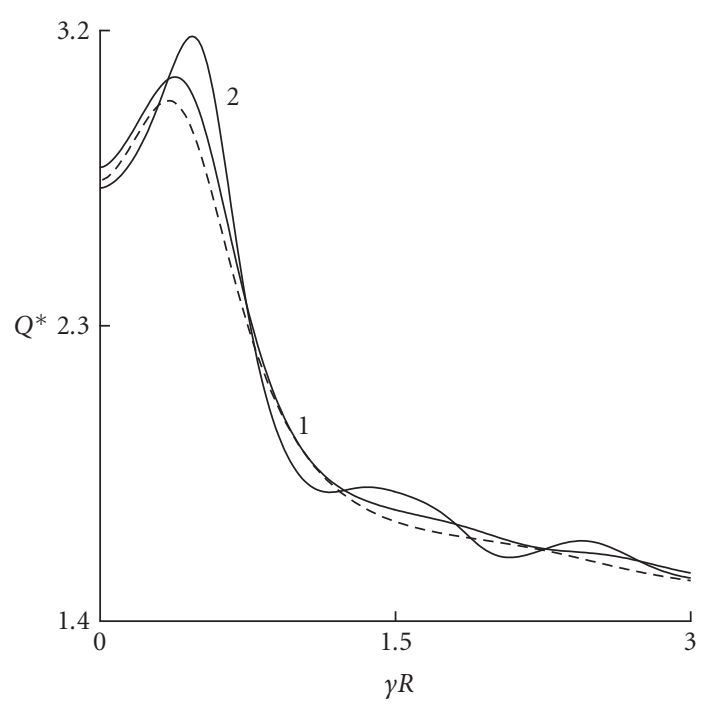

Figure 6.1. Changes of a relative total electric charge on an electrode in the function of the normalized wave number $\left(h_{1} / R_{1}=3\right)$.

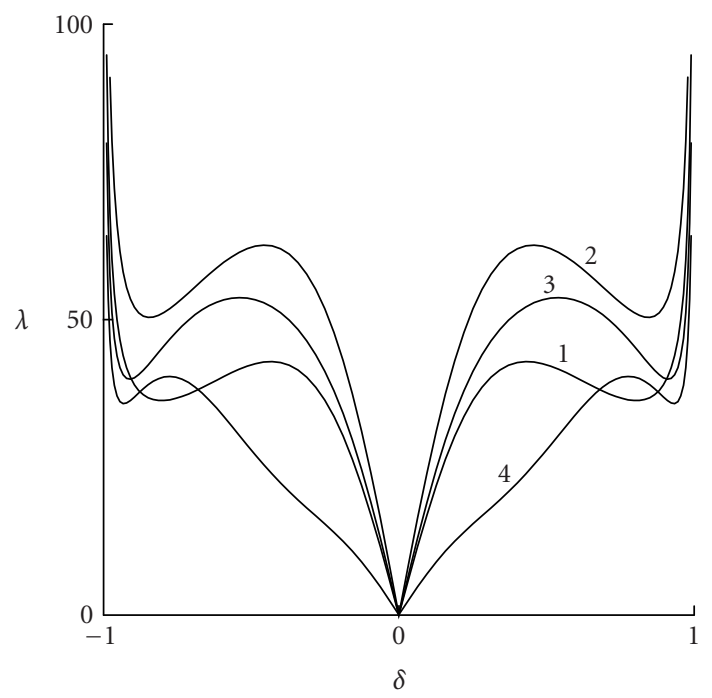

Figure 6.2. Changes of intensity of contact forces on a linear insert for various values of the normalized wave number $\gamma R$.

of the displacement amplitude in the area covering a circular tunnel opening and linear stringer are represented in Figure $6.6\left(\beta_{1}=5 \pi / 14, \beta_{2}=9 \pi / 14, \beta_{3}=19 \pi / 14, \beta_{4}=\right.$ $23 \pi / 14)$ and Figure $6.7\left(\beta_{1}=\pi / 6, \beta_{2}=5 \pi / 6, \beta_{3}=7 \pi / 6, \beta_{4}=11 \pi / 6\right)$ at $p_{1} / R=2, h / R=$ $4, \gamma R=1$. The more light areas correspond to the maximum values of quantity $\left|U_{3}\right|$. 


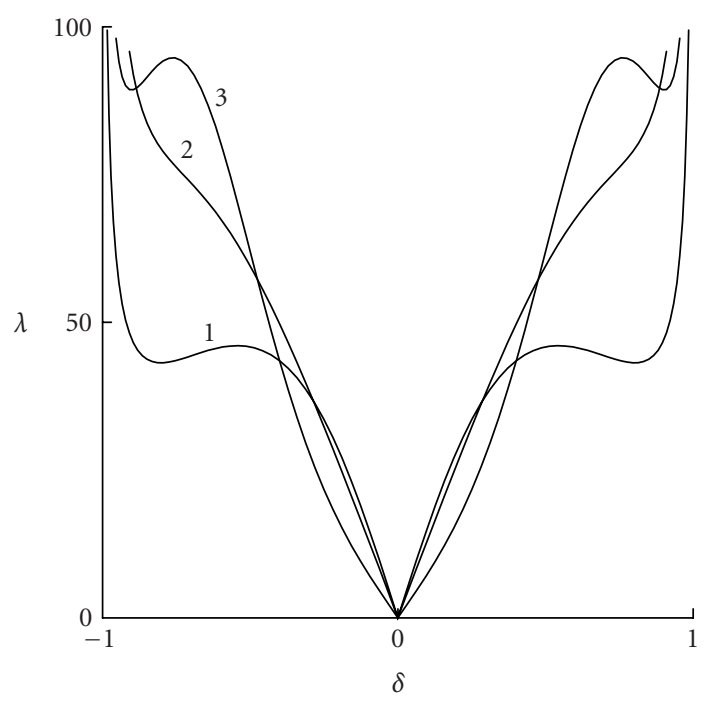

Figure 6.3. Changes of intensity of contact forces on a parabolic insert for various values of the curvature parameter.

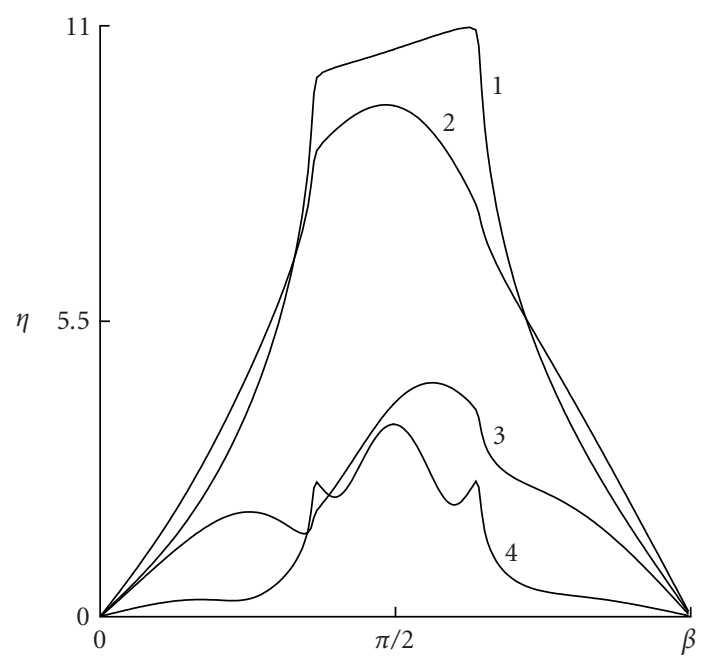

Figure 6.4. Changes of the modulus of displacement amplitude on the contour of an elliptic opening with two electrodes for various values $\gamma R$.

Figure 6.8 illustrates the lines of level $\left|U_{3}\right|$ in the vicinity of elliptic opening $\left(R_{1} / R_{2}=2\right)$ and of a stringer for the values of the parameters corresponding to Figure 6.6 and $\gamma R=2$.

\section{Conclusions}

From the given results it is shown that the availability of an inclusion may increase the influence of the dynamic effect on the behavior of total charges on electrodes. For example, 


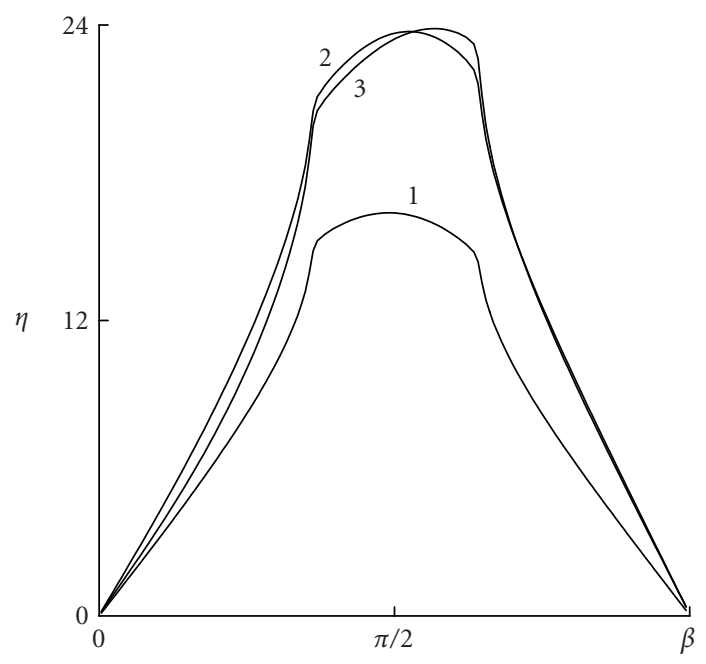

Figure 6.5. Changes of the modulus of displacement amplitude on the contour of an elliptic opening with two electrodes for various values of the curvature parameters.

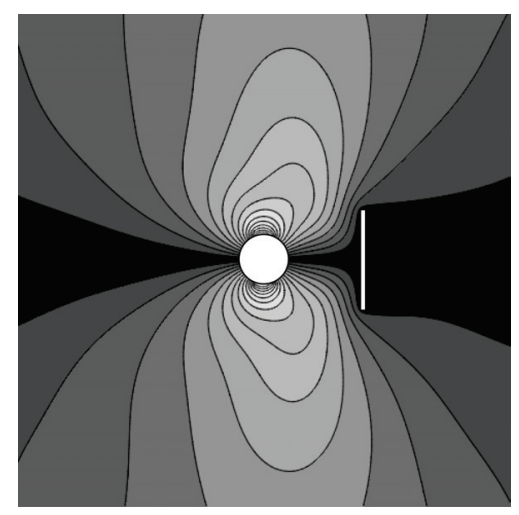

Figure 6.6. The level lines of the modulus of displacement amplitude in a medium with heterogeneities $\left(\gamma R=1, \beta_{1}=5 \pi / 14, \beta_{2}=9 \pi / 14, \beta_{3}=19 \pi / 14, \beta_{4}=23 \pi / 14\right)$.

as it follows from Figure 6.1, quantity $Q^{*}$ may excess its static analogue by $17 \%$ (curve 2 ). In the absence of the inclusion the dynamic effect is only equal to $9 \%$ [2]. As it follows from Figures 6.6, 6.7, and 6.8, a fixed rigid stringer impedes the distribution of the medium oscillation.

Also, we should note here that according to the frequency of excitation, the stringer configuration, and the sizes of electrodized areas, there occur redistribution of contact forces on the insert contour and displacements on the opening boundary. At antiplane deformation the stresses of a longitudinal shear on a free-from-mechanical-loading surface do not have singularities on the edges of the electrodes. The numerical calculations 


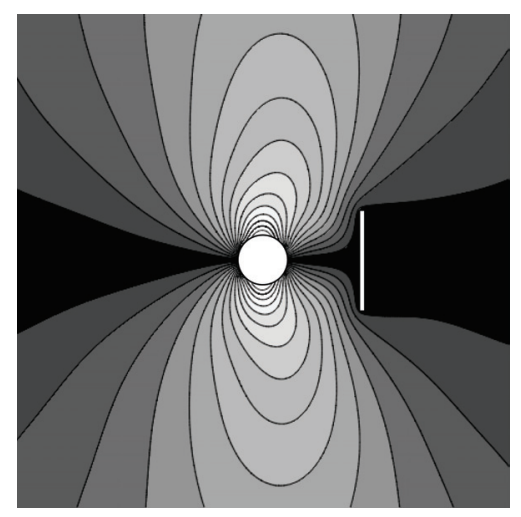

Figure 6.7. The level lines of the modulus of displacement amplitude in a medium with heterogeneities $\left(\gamma R=1, \beta_{1}=\pi / 6, \beta_{2}=5 \pi / 6, \beta_{3}=7 \pi / 6, \beta_{4}=11 \pi / 6\right)$.

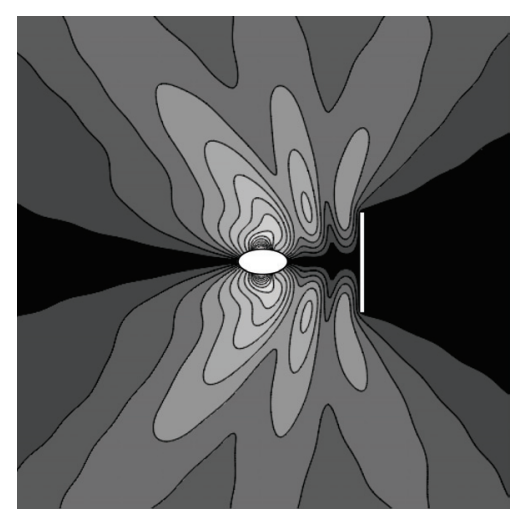

Figure 6.8. The level lines of the modulus of displacement amplitude in a medium with an elliptic opening and inclusion $\left(\gamma R=2, \beta_{1}=5 \pi / 14, \beta_{2}=9 \pi / 14, \beta_{3}=19 \pi / 14, \beta_{4}=23 \pi / 14\right)$.

proceeding from the here constructed algorithm and formula (5.2) confirm this conclusion. However, the components of the vector of electric induction possess the singularities of root type on the edges of the electrodes, what directly follows from the consideration of singular integral equations in (3.2) and expressions (4.6), (4.7).

\section{References}

[1] D. Bardzokas, G. E. Exadaktylos, and G. Anastaselos, The effect of stringers and patches on the stress intensities around cracks in plates, Engrg. Fracture Mech. 55 (1996), no. 6, 935-955.

[2] D. Bardzokas and M. L. Filshtinsky, Electroelasticity of Piecewise-Uniform Bodies, Ukraine: University Book Publishing, Sumy, 2000.

[3] D. Bardzokas, B. A. Kudryavtsev, and N. A. Senik, On criteria of electromechanical failure of piezoelectrics initiated by the edges of electrodes, Strength of Materials International journal (1994), no. 7, 42-46. 


\section{Excitation of shear waves in a piezoceramic medium}

[4] , The Rayleigh waves in a half-space with a finite system of surface electrodes, Mech. Solids (1996), no. 1, 45-51 (Russian).

[5] D. A. Berlincourt, D. R. Curran, and H. Jaffe, Piezoelectric and piezomagnetic materials and their functions as transducers, Physical Acoustics, V.1, Part A. (W. P. Mason, ed.), Academic Press, New-York, 1964.

[6] M. L. Filshtinsky, On an approach to the investigation of electroelastic fields in a cylinder excited by the system of surface electrodes, no. 1(9), Proceedings of Sumy University, Sumy, 1998, pp. 3-8 (Russian).

[7] B. A. Kudryavtsev, Electroelastic state of a half-plane from piezoceramics with two boundary electrodes, Strength of Materials International journal (1982), no. 7, 56-59.

[8] G. A. Maugin, Continuum Mechanics of Electromagnetic Solids, North-Holland Series in Applied Mathematics and Mechanics, vol. 33, North-Holland Publishing company, Amsterdam, 1988.

[9] N. I. Muskhelishvili, Singular Integral Equations, Wolters-Noordhoff Publishing, Groningen, 1958.

[10] W. Nowacki, Electromagnetic Effects in Solids, Mechanics: Recent Publications in Foreign Science, Vol. 37, Mir, Moscow, 1986 (Russian).

[11] V. Z. Parton and B. A. Kudryavtsev, Electromagnetoelasticity, Gordon and Breach, New York, 1988.

[12] P. S. Theocaris and D. Bardzokas, The influence of a finite stringer on the stress intensities around cracks in plates, Engrg. Fracture Mech. 14 (1981), 493-506.

D. I. Bardzokas: Laboratory of Strength and Materials, Department of Mechanics, Faculty of Applied Sciences, National Technical University of Athens, Zografou Campus, Theocaris Building, 15773 Athens, Greece

E-mail address: bardim@central.ntua.gr

G. I. Sfyris: Laboratory of Strength and Materials, Department of Mechanics, Faculty of Applied Sciences, National Technical University of Athens, Zografou Campus, Theocaris Building, 15773 Athens, Greece

E-mail address: gsfyris@mail.ntua.gr 


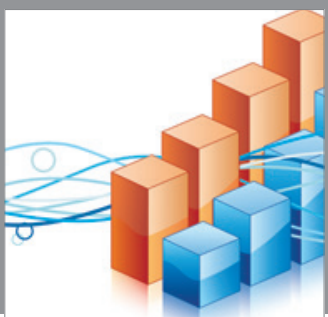

Advances in

Operations Research

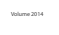

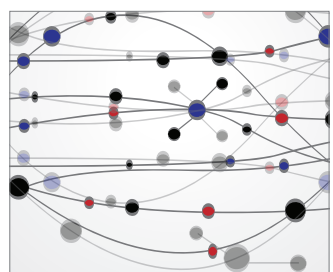

\section{The Scientific} World Journal
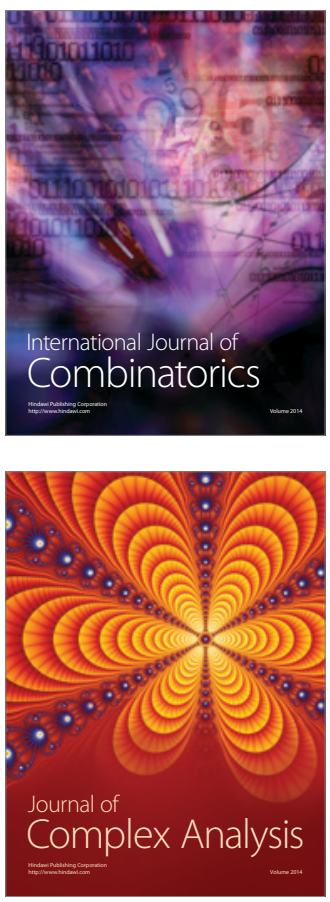

International Journal of

Mathematics and

Mathematical

Sciences
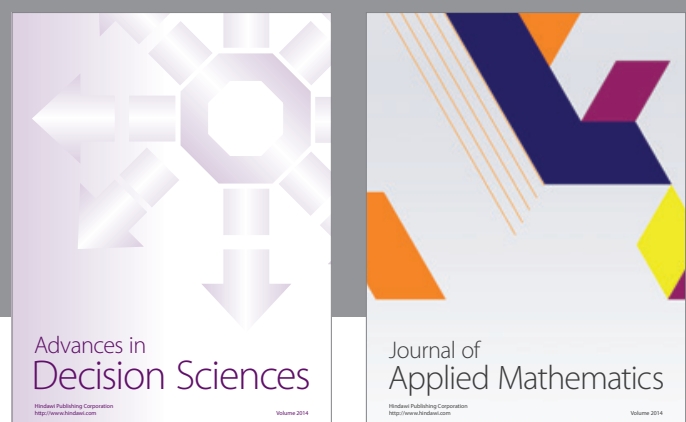

Journal of

Applied Mathematics
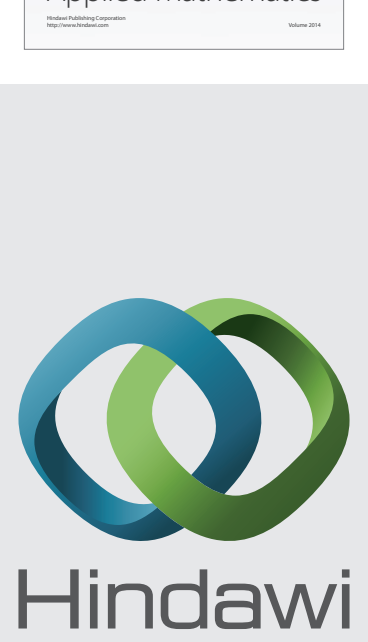

Submit your manuscripts at http://www.hindawi.com
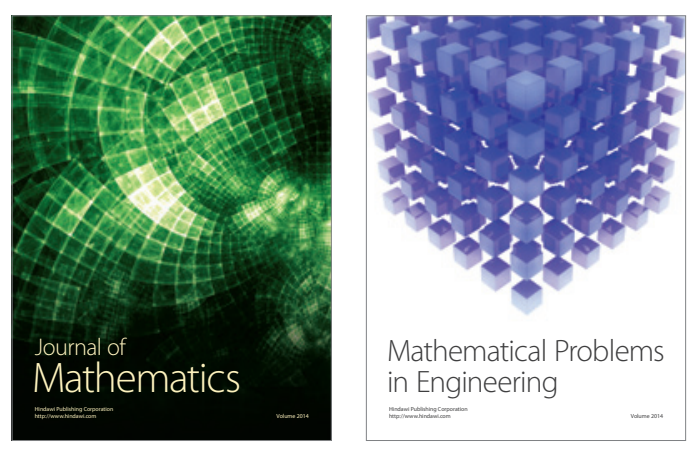

Mathematical Problems in Engineering
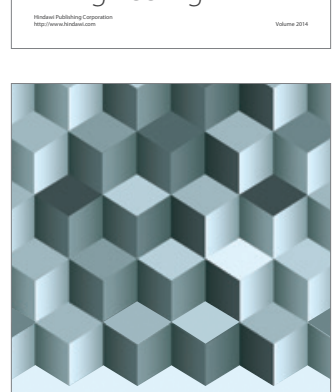

Journal of

Function Spaces
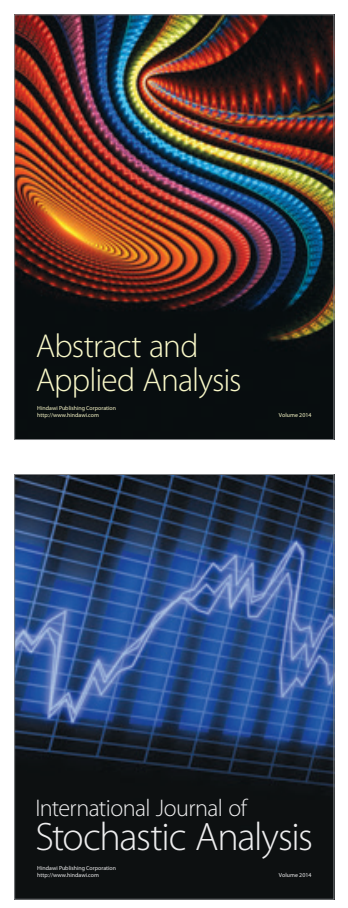

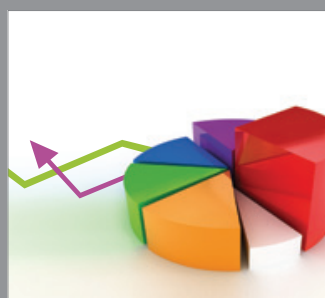

ournal of

Probability and Statistics

Promensencen
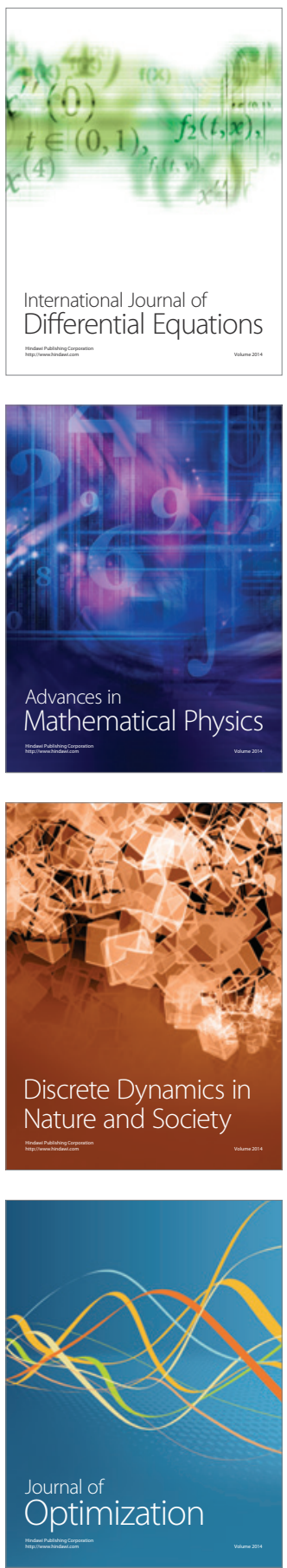